\title{
Four Different Subunits Are Essential for Expressing the Synaptic Glutamate Receptor at Neuromuscular Junctions of Drosophila
}

\author{
Gang Qin, ${ }^{1}$ Tobias Schwarz, ${ }^{1}$ Robert J. Kittel, ${ }^{1}$ Andreas Schmid, ${ }^{1}$ Tobias M. Rasse, ${ }^{1}$ Dennis Kappei, ${ }^{1}$ \\ Evgeni Ponimaskin, ${ }^{2}$ Manfred Heckmann, ${ }^{3}$ and Stephan J. Sigrist ${ }^{1}$ \\ ${ }^{1}$ European Neuroscience Institute Göttingen, Max-Planck-Society, D-37073 Göttingen, Germany, ${ }^{2}$ Departments of Neurophysiology and Sensory \\ Physiology, Medical School at the University of Göttingen, D-37073 Göttingen, Germany, and ${ }^{3}$ Physiologisches Institut, D-79104 Freiburg, Germany
}

\begin{abstract}
Three ionotropic glutamate receptor subunits, designated GluRIIA, GluRIIB, and GluRIII, have been identified at neuromuscular junctions of Drosophila. Whereas GluRIIA and GluRIIB are redundant for viability, it was shown recently that GluRIII is essential for both the synaptic localization of GluRIIA and GluRIIB and the viability of Drosophila. Here we identify a fourth and a fifth subunit expressed in the neuromuscular system, which we name GluRIID and GluRIIE. Both new subunits we show to be necessary for survival. Moreover, both GluRIID and GluRIIE are required for the synaptic expression of all other glutamate receptor subunits. All five subunits are interdependent for receptor function, synaptic receptor expression, and viability. This indicates that synaptic glutamate receptors incorporate the GluRIII, GluRIID, and GluRIIE subunit together with either GluRIIA or GluRIIB at the Drosophila neuromuscular junction. At this widely used model synapse, the assembly of four different subunits to form an individual glutamate receptor channel may thus be obligatory. This study opens the way for a further characterization of in vivo glutamate receptor assembly and trafficking using the efficient genetics of Drosophila.
\end{abstract}

Key words: Drosophila; synapse; neurotransmission; glutamate receptor; subunit composition; neuromuscular junction

\section{Introduction}

Ionotropic glutamate receptors (GluRs) mediate most excitatory synaptic transmission in our nervous system. One subgroup of glutamate receptors, the so-called non-NMDA-type glutamate receptor channels, preferentially mediate fast synaptic transmission (Dingledine et al., 1999).

Ionotropic glutamate receptors are supposed to be composed of four subunits in total (Laube et al., 1998; Mano and Teichberg, 1998; Rosenmund et al., 1998). The functional properties of individual non-NMDA receptors, such as single channel conductance, $\mathrm{Ca}^{2+}$ permeability, and rectification, are subunit dependent and can be controlled by alternative splicing and RNA editing at key positions of individual subunits (Seeburg, 1993; Mayer and Armstrong, 2004). In addition, subunit composition is important for the assembly, trafficking, insertion, synaptic localization, and anchoring of non-NMDA receptors in synapses (Malinow and Malenka, 2002; Bredt and Nicoll, 2003). Many ligand-gated receptor channels, such as the glycine receptor and the nicotinic acetylcholine receptor, have been shown to have fixed subunit stoichiometries (Colquhoun and Sivilotti, 2004).

Received 0ct. 8, 2004; revised Jan. 22, 2005; accepted Feb. 14, 2005

This work was supported by grants from the Deutsche Forschungsgemeinschaft (S.J.S.). We thank Dave Featherstone and Kendal Broadie for sharing unpublished data and helpful discussions and Aaron DiAntonio for fly lines. Correspondence should be addressed to Stephan J. Sigrist, Max-Planck-Society, European Neuroscience Institute, Waldweg 33, D-37073 Göttingen, Germany. E-mail: ssigris@gwdg.de.

DOI:10.1523/JNEUROSCI.4194-04.2005

Copyright $\odot 2005$ Society for Neuroscience $\quad$ 0270-6474/05/253209-10\$15.00/0
Nevertheless, initial work implied that non-NMDA glutamate receptors could consist of various subunit combinations. However, assembly of non-NMDA receptors in mammals is assumed to be restricted in that an individual non-NMDA receptor is composed of no more than two different subunits (Ayalon and SternBach, 2001; Mansour et al., 2001; Sun et al., 2002; Schorge and Colquhoun, 2003). Although elegant work using functional and/or optical subunit labeling has enabled the study of assembly mode, trafficking, insertion, and anchoring of individual nonNMDA receptors (for review, see Malinow and Malenka, 2002), the subunit composition of entire native receptors still remains difficult to address under physiological conditions at synapses.

Here, we use the glutamatergic neuromuscular junction (NMJ) of Drosophila to explore the subunit composition of a synaptic non-NMDA glutamate receptor in vivo. This synapse is widely used for functional studies of synaptic transmission (Bellen, 1998; Gramates and Budnik, 1999; Featherstone and Broadie, 2000; Koh et al., 2000) and expresses postsynaptic glutamate receptors that are homologous to vertebrate non-NMDA glutamate receptors (Schuster et al., 1991; DiAntonio et al., 1999; Marrus et al., 2004). Subunit composition has been shown to both regulate biophysical properties of glutamate receptors and to control synapse formation and function in this model system (DiAntonio et al., 1999; Sigrist et al., 2000, 2002). Our goal was to turn this established experimental preparation into a model system in which the subunit composition of the postsynaptic nonNMDA glutamate receptors can be fully controlled. Therefore, 
we searched for additional essential non-NMDA receptors at the neuromuscular junction of Drosophila. We can identify two additional neuromuscular glutamate receptor subunits, GluRIID and GluRIIE, which are essential for viability. GluRIID can be shown to be expressed within the postsynaptic densities (PSDs) of all NMJs. Knock-out and rescue experiments with the five total subunits were performed to address the in vivo subunit composition of the synaptic glutamate receptors in this preparation. Our data suggest that, here, individual synaptic glutamate receptor channels may contain not only two but four different subunits.

\section{Materials and Methods}

\section{Microarray analysis and quantitative real-time PCR}

Microarray experiments were performed using Affymetrix (Santa Clara, CA) Drosophila GeneChips and standard protocols as described in the Affymetrix gene chip user manual. RNA was isolated from whole animal or larval body-wall preparations (epidermis with body-wall muscles attached) of midstage third-instar wild-type larvae (CS10) reared at $25^{\circ} \mathrm{C}$. Biotinylated cRNAs were prepared from double-stranded cDNA pools and used to probe Affymetrix high-density oligonucleotide arrays. Hybridization, staining, and washing were done according to the instructions in the user manual. Data analysis was performed via Microarray Suite 4.0 (Affymetrix).

For real-time PCR-based mRNA quantification, the same RNA samples as also used for chip analysis were reverse transcribed and PCR amplified by Omniscript RT kit and QuantiTect SYBY Green PCR kit (Qiagen, Hilden, Germany). Primers were designed via PrimerExpress version 2.0 (PE Applied Biosystems, Foster City, CA). Primers used were as follows: $t b p-1$ (TaT binding protein-1), $5^{\prime}$ AAGCCCGTGCCCGTATTATG3' and 5'AAGTCATCCGTGGATCGGGAC3'; GluRIIA, 5'CCCAGATTGGCGAGCAGAT3' and 5'CCGGTAATCAGAGCCCAGTG3'; GluRIIB, 5'GATGATGGCCAGTTCGACATG3' and 5'TCAGCACCACGAACAGTCCA3'; GluRIIC/III, 5'CCTCCATCATGACAGCAGGA3' and 5'GCACCTGTGGACTTCTCGGT3'; GluRIID, 5'ACGTCATCGAACTGCAAACCA3' and 5'TCGCTGGAACTCGAAGTCCTT3'; and GluRIIE, 5'TGGCTGCCTTTTTGACCATC3' and 5'ACCATCCTTGTTATCGGCCAG3'.

Real-time quantitative PCR was performed in GeneAmp 5700 Sequence Detection System (PE Applied Biosystems), and data were analyzed with Excel (Microsoft, Seattle, WA).

\section{Genetics and molecular biology}

GluRIID and GluRIIE genetics. The GluRIID and GluRIIE loci are situated at 92F4. In a recently released pBac transposon insertion collection (Thibault et al., 2004), a piggyBac transposon (pBac\{RB\}e01443) was found to be inserted into intron 6 at amino acid position 427 of the GluRIID open reading frame. This allele GluRIID ${ }^{\text {eOI443 }}$ is embryonic lethal both homozygous and over $d f(3 R) H-B 79$ (Bloomington Drosophila stock center), which deletes a large genomic region including the GluRIID and GluRIIE locus, and over deficiency GluRIID $I_{I I E}{ }^{E 3}$ (see below). For GluRIIE, imprecise excision screening was performed using the P-element line EP28753 (commercially available with Genexcel), which is inserted $\sim 150$ bp downstream of the end of the GluRIIE transcript. In brief, P-element EP28753 was remobilized by crossing to the $\Delta 2-3$ transposase source, white eye progenies were selected and mated individually, and then single fly genomic PCR reactions were performed to map deletions flanking the P-element insertion site. Nearly 1000 eye color revertants were checked, and one line $\left(G l u R I I E^{E I}\right)$ was found to delete $1.2 \mathrm{~kb}$ flanking region in direction of the GluRIIE gene, removing the C-terminal and transmembrane domain 4 of GluRIIE. GluRIIE ${ }^{E 1}$ is embryonic lethal when homozygous, over $d f(3 R) H-B 79$ and over GluRIID\&IIEE3. GluRIID\&IIE ${ }^{E 3}$ is a larger deletion also recovered from excision mutagenesis of EP28753, which removes both GluRIIE and GluRIID and thus was used in trans to either GluRIID ${ }^{e 01443}$ or GluRIIE EI deficiency to study GluRIID and GluRIIE null mutant situations.

Double mutants in GluRIIA and GluRIIB were described previously (Petersen et al., 1997; DiAntonio et al., 1999). In short, GluRIIA and GluRIIB double-mutant embryos were recovered by crossing $d f(2 L)$ GluRIIA $B^{S P 22}$ to $d f(2 L) c l^{h 4}$, and mutant embryos were selected using green fluorescent protein (GFP)-marked balancer chromosomes. GluRIIA $^{\text {hypo }}$, GluRIIB ${ }^{\text {null }}$ larvae have an extremely reduced amount of GluRIIA and no GluRIIB expression. For this, a genomic fragment of GluRIIA, encompassing promoter region and the whole open reading frame but missing most of the $3^{\prime}$ untranslated region ( $3^{\prime}$-UTR), was used. This transgene still produces full-length GluRIIA while in dramatically reduced amounts because of a loss of message stability (G. Qin and S. Sigrist, unpublished observations). This construct was expressed from pUAST (using the GluRIIA endogenous promoter). A single transgene copy rescues embryos null for both GluRIIA and GluRIIB $\left[d f(2 L) G l u R I I A \& B^{S P 22} / d f(2 L) c l^{h 4}\right]$, giving GluRIIA hypo,$G l u R I I B^{\text {null }}$ larvae (see Results). The genomic fragment with $3^{\prime}$-UTR deletion was produced as follows: an EcoRI/XhoI fragment $(5.6 \mathrm{~kb})$ from BAC clone RPCI-98-35L07 containing the GluRIIA gene including $1.3 \mathrm{~kb}$ sequence $5^{\prime}$ of the ATG was subcloned into pSL1180. Inserting this EcoRI/XhoI fragment into pUAST gave a "wild-type GluRIIA rescue construct." For the 3'-UTR-deleted version, this clone was cut with $\mathrm{NcoI}$ and XhoI, end blunted, and religated, and then an EcoRI/Asp718 fragment ( $4.7 \mathrm{~kb})$ was inserted into pUAST. All constructs were confirmed by double-strand sequencing.

Transgenic rescue constructs. Genomic fragments covering the GluRIID and GluRIIE genes were generated by PCR using for IID 5'GGTCTAGAGCGGCCGCGGCCACGAACTGACCCACGGTTTC3' and 5'GCGGCCCTCGAGCGACGTCAAGGATGTGCCCAC3' and for IIE 5'GGTCTAGAGCGGCCGCACCTCCCCAAGCTGTCAACTTC3' and 5'GCGGCCCTCGAGACTGCTCAAAGCTGCTGCCCTG3'. The products were double-strand sequenced and cloned into pUAST. Several independent lines of transgenic animals were generated. For overexpression studies, UAS-GluRIID and UAS-GluRIIE were generated by introducing the full-length cDNA into the transformation vector pUAST. Full-length cDNAs of GluRIID (RE24732) and GluRIIE (RE07945) were obtained from Berkeley Drosophila Genome Project (Berkeley, CA) cDNA libraries.

The construct for inducible RNA interference (RNAi-GluRIIE) was made based on the pUASTi plasmid (contains an intron between insertion sites for sense and antisense fragments; generous gift from Amin Ghabrial, Krasnow laboratory, Stanford University, Stanford, CA). Selected cDNA fragments coving part of $5^{\prime}$-UTR and coding region were PCR amplified by using the following primer pairs: 5'GCGCGCCTCGAGCTGTTCGGGAAACTCAAGAAT3' and 5'GGTCTAGAGCGGCCGCCGTGGTTAGCTCGTTCAAAATG3' for fragment 1 (sense); and 5'GCTGGTACCTGTTCGGGAAACTCAAGAAT3' and 5'GCGTCTAGATCGTGGTTAGCTCGTTCAAAATG3' for fragment 2 (antisense).

The two fragments were inserted into pUASTi plasmid sequentially and verified by sequencing. Several independent lines of transgenic animals carrying UAS-GluRIIE were generated.

To express GluRIIE-GFP in SF9 cells and flies, a HindIII fragment covering the enhanced GFP (EGFP) insertion site (position 880 of GluRIIE) was cut from full-length GluRIIE cDNA (RE07945) and subcloned into $p S L 1180$. On this vector, "circular PCR" with primers 5'GGCAGATGTGTATAAGAGACAGTCGCCAGTCCTCGATGTCAGTAGCTT3' and 5'CGGGCGCGCCGCCCTTGTACAGCTCGTCCATGCCGAGA3' was performed, and the resulting fragment was bluntend ligated with an EGFP encoding fragment, which was PCR amplified using 5'GGCGCGCCGAGCAAGGGCGAGGAGCTGTTCACCGG3' and 5'CGGGCGCGCCGCCCTTGTACAGCTCGTCCATGCCGAGA3' from a EGFP containing vector (Clontech, Cambridge, UK). Correct orientation of EGFP was verified by PCR and sequencing. Then the HindIII fragment containing EGFP sequence was put back into the fulllength cDNA clone, and the reading frame was verified by PCR and sequencing. Finally, the full-length GluRIIE cDNA tagged with EGFP was transferred into pFastBac (Invitrogen, San Diego, CA). Baculovirus expression was performed as described previously (Swan et al., 2004) or into $p U A S T$ for transgenesis.

\section{Stainings}

In situ hybridization. Whole-mount embryonic and larval body-wall preparation in situ hybridizations were performed essentially as de- 
Table 1. GluRIID and GluRIIE are enriched in larval body-wall mRNA

\begin{tabular}{llllll}
\hline & $C_{\mathrm{t}}$ (body wall) & \% Control & $C_{\mathrm{t}}$ (total larva) & \% Control & Body wall/total larva \\
\hline GluRIIA (CG6992) & 26.7 & 15.4 & 28.3 & 4.4 & 3.5 \\
GluRIIB (CG7234) & 28.2 & 5.6 & 29.5 & 1.9 & 3.0 \\
GluRIIC/III (CG4226) & 26.4 & 18.7 & 28.9 & 3.1 & 6.3 \\
GluRIID (CG18039) & 25.5 & 35.5 & 27.0 & 10.5 & 3.4 \\
GluRIIE (CG31201) & 24.5 & 73.7 & 26.3 & 17.5 & 4.2 \\
tbp (control) & 23.8 & 100.0 & 23.8 & 100.0 & 1.0 \\
\hline
\end{tabular}

Abundances of glutamate receptor subunit transcripts estimated using real-time PCR. The control transcript (proteasome subunit tbp-1) had the same abundance in body-wall preparations and whole larvae. The glutamate receptor subunits shown were enriched in body-wall RNA when compared with whole larva RNA. The abundances of mRNAs are expressed as $C_{\mathrm{t}}$ values, indicating the cycle number with which amplification exceeds detection threshold $(C$ difference of 1 indicates a twofold difference in abundance). For all reactions, identical amounts of cDNA were used. All data are averages from three independent experiments, in which each sample was run three times in parallel.

scribed previously (Tautz and Pfeifle, 1989). For preparing antisense RNA probes, both RE24732 (GluRIID) and RE07945 (GluRIIE) plasmids were cut with NotI and in vitro transcribed using T3 RNA polymerase.

Immunohistochemistry. Rabbit anti-GluRIIC/III antibodies were generated against a C-terminal close peptide of GluRIIC/III (PRRSLDKSLDRTPKS). Rabbit anti-GluRIID antibodies were generated against a C-terminal peptide of GluRIID (ESLKTDSEENMPVED). Both sera were affinity purified and used at 1:500 dilution. Other primary antibodies were used at the following concentrations: mouse monoclonal antiGluRIIA antibody (8B4D2; Developmental Studies Hybridoma Bank, University of Iowa, Iowa City, IA), 1:100; goat anti-HRP cyanine 5, 1:250; mouse anti-FasII (1D4; Developmental Studies Hybridoma Bank), 1:40; mouse anti-Discs Large (Developmental Studies Hybridoma Bank), 1:500; Nc82 (generous gift from Erich Buchner, University of Würzburg, Würzburg, Germany), 1:100; rabbit anti-GFP (Molecular Probes, Eugene, OR), 1:300. Except for samples stained with 8B4D2, which were fixed for $5 \mathrm{~min}$ with cold methanol, all of the other stainings were fixed for $10 \mathrm{~min}$ with $4 \%$ paraformaldehyde.

Dissection and immunostaining. Midstage third-instar larvae were put on a dissection plate with both ends fixed by fine pins and then covered by a drop of ice-cold hemolymph-like saline (HL-3) solution (see below). Dissection scissors were used to make a small hole at the dorsal midline of the larva (near to the posterior end), which was then completely opened along the dorsal midline from the hole to the anterior end. The epidermis was stretched flat and pinned down, and then the internal organs and CNS were removed carefully with forceps. Late-stage embryos (20-22 h after egg laying) were dissected on sylgard plates, fixed with fine clips, and opened using a pair of sharp tungsten needles. The dissected samples were fixed and then incubated with primary antibodies overnight, followed by fluorescence-labeled secondary antibodies (Dianova, Hamburg, Germany) and mounted in VectaShield mounting media (Vector Laboratories, Burlingame, CA).

Imaging and analysis. Imaging of embryonic and larval body-wall preparations was performed on a Leica (Nussloch, Germany) DM IRE2 microscope equipped with a Leica TCS SP2 AOBS scan head, using a Leica HCX PL Apo CS 63×, 1.32 numerical aperture oil ultraviolet objective. Image processing was performed using NIH ImageJ and Photoshop (Adobe Systems, San Jose, CA). Epifluorescence images were taken on a Zeiss (Oberkochen, Germany) Axioscope with Axiocam camera, using a $100 \times$ oil objective of numerical aperture 1.4.

\section{Electrophysiology}

Intracellular recordings were made at $22^{\circ} \mathrm{C}$ from muscle fiber 6 of abdominal segments 2 and 3 of late third-instar larvae. Larvae were dissected in ice-cold, calcium-free hemolymph-like saline (HL-3) (Stewart et al., 1994). Composition of the HL-3 solution was as follows (in mM): 70 $\mathrm{NaCl}, 5 \mathrm{KCl}, 20 \mathrm{MgCl}_{2}, 10 \mathrm{NaHCO}_{3}, 5$ trehalose, 115 sucrose, and 5 HEPES, $\mathrm{pH}$ adjusted to 7.2. Larval fillets were rinsed with $2 \mathrm{ml}$ of HL-3 saline containing $1 \mathrm{mM} \mathrm{Ca}^{2+}$ before being transferred to the recording chamber in which two-electrode voltage-clamp (TEVC) recordings were performed in $1 \mathrm{~mm}$ extracellular $\mathrm{Ca}^{2+}$. The larval NMJ was visualized with a fixed-stage upright microscope $(40 \times$ water immersion lens; Olympus Optical, Tokyo, Japan). Whole muscle recordings of both miniature and evoked postsynaptic currents were recorded in TEVC mode (AxoClamp 2B; Axon Instruments, Union City, CA) using sharp micro- electrodes (borosilicate glass with filament, 1.5 mm outer diameter) with resistances of 15-35 $\mathrm{M} \Omega$ and filled with $3 \mathrm{M}$ KCL. All cells selected for analysis had resting potentials between -60 and $-70 \mathrm{mV}$. For stimulation, the cut end of the segmental nerve was pulled into a firepolished suction electrode, and brief $(300 \mu \mathrm{s})$ depolarizing pulses were passed at $0.2 \mathrm{~Hz}$ [NPI Electronics (Tamm, Germany) stimulus generator and isolation unit]. To ensure the stable recruitment of both innervating motoneurons, the amplitude of the pulse was determined by increasing the stimulation strength to 1.5 times the amplitude needed to reach the threshold of double-motoneuron recruitment. The clamp was tuned such that it responded to a voltage step from -60 to $-70 \mathrm{mV}$ with settling times of $1 \mathrm{~ms}$ for miniature excitatory junctional currents (mEJCs) and 500-750 $\mu$ s for evoked EJCs (eEJCs); this gave voltage errors of maximally $4 \mathrm{mV}$ for eEJCs of approximately $-100 \mathrm{nA}$. Both eEJCs (voltage clamp at $-60 \mathrm{mV}$ ) and mEJCs (voltage clamp at $-80 \mathrm{mV}$ ) were low-pass filtered at $1 \mathrm{kHz}$, and the holding current never exceeded $-10 \mathrm{nA}$. For each cell, 20 eEJCs and $90 \mathrm{~s}$ of mEJC recordings were used for subsequent analysis (pClamp9; Axon Instruments).

\section{Results}

\section{New glutamate receptor subunits expressed within postsynaptic muscle cells of Drosophila}

The Drosophila genome encodes $\sim 30$ potential glutamate receptor subunits (Littleton and Ganetzky, 2000; Sprengel et al., 2001). We explored how many glutamate receptor subunits might be expressed in total within the postsynaptic muscle cell. To this end, RNA was extracted from larval body-wall preparations, which are enriched for somatic muscles but free of CNS tissue and internal organs. Using Affymetrix Drosophila gene chips, the abundances of Drosophila glutamate receptor subunits were determined in larval body-wall RNA. The amount of each subunit was then compared with the abundance in whole larval mRNA. As expected, GluRIIA and GluRIIB (Schuster et al., 1991; Petersen et al., 1997) were found to be enriched in body-wall RNA. Also, the subunit annotated as CG4226, first referred to as GluRIIC (Saitoe et al., 2001; Sprengel et al., 2001) and later as GluRIII (Marrus et al., 2004), was found to be enriched. Recent work had shown that GluRIII mutant embryos die, most likely because of a defect of glutamatergic transmission. In addition to these already described subunits with NMJ expression, the mRNA of another locus encoding a glutamate receptor subunit (annotated as CG18039) was also enriched in body-wall preparations.

Next, real-time PCR was used to independently quantify the expression of these glutamate receptor subunits. Real-time PCR confirmed the body-wall enrichments first observed by gene chip analysis (Table 1). We further recognized that CG31201, the direct neighbor locus of CG18039, encodes a glutamate receptor as well. This locus was not represented on the chip used. We also found CG31201 to be enriched within body-wall mRNA (Table $1)$. From now on, we will refer to the locus encoding subunit CG18039 as GluRIID and the locus encoding CG31021 as GluRIIE. These names are meant to reflect muscle expression (see below) along with GluRIIA, GluRIIB, and GluRIIC/III.

To confirm muscle expression of these new subunits, in situ hybridization on Drosophila embryos and larvae was performed. In fact, the mRNAs of GluRIID (Fig. 1A-D) and GluRIIE (Fig. $1 E, F)$ are enriched in somatic muscles of both the Drosophila embryo (Fig. 1A-C,E,F) and larva (Fig. 1D). Expression of GluRIID (Fig. $1 C-D$ ) and GluRIIE (Fig. $1 E$ ) starts in somatic muscles of late-stage 12 embryos (Fig. 1A,E) and extends 


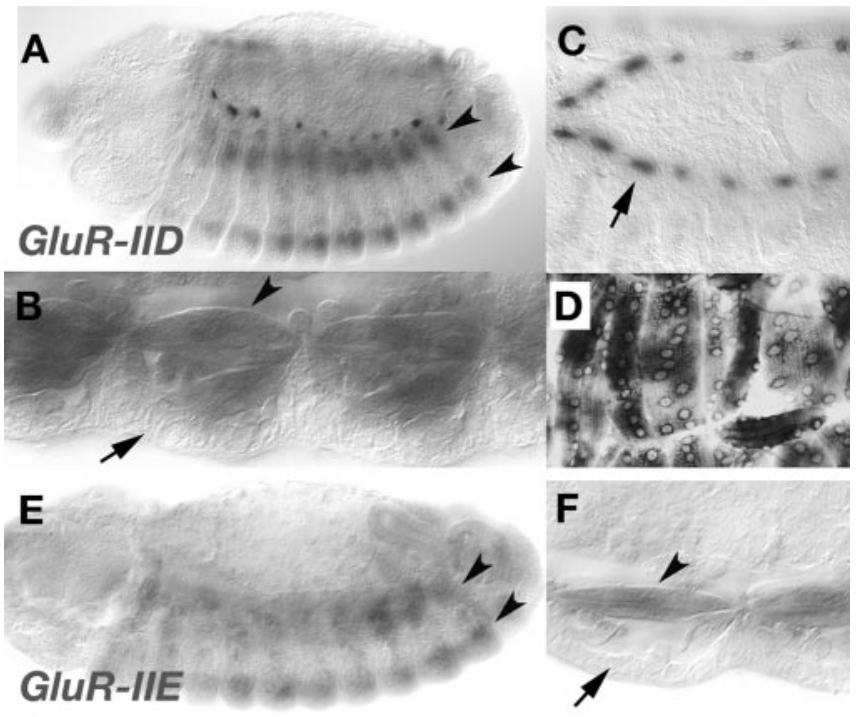

Figure 1. GluRIID and GluRIIE: novel glutamate receptor subunits with muscle-specific expression. In situ hybridizations on Drosophila embryos $(\boldsymbol{A}-\boldsymbol{C}, \boldsymbol{E}, \boldsymbol{F})$ and larvae $(\boldsymbol{D})$ for GluRIID $(\boldsymbol{A}-\boldsymbol{D})$ and GluRIIE $(\boldsymbol{E}, \boldsymbol{F})$ mRNA. Both subunits are strongly enriched in presumptive somatic muscle cells ( $\boldsymbol{A}, \boldsymbol{B}, \boldsymbol{E}, \boldsymbol{F}$, arrowheads) but are, for example, not found in the adjacent epidermis $(\boldsymbol{A}, \boldsymbol{B}, \boldsymbol{F}$, arrows). Expression of GluRIID and GluRIIE transcript in the presumptive somatic muscles starts in late stage 12 and peaks at approximately stage $14(\boldsymbol{A}, \boldsymbol{E})$, to then persist during later embryogenesis ( $\boldsymbol{B}$, stage $16 ; \boldsymbol{E}$, stage 17$)$ and larval development (D). GluRIID is also expressed within heart precursor cells ( $\boldsymbol{A}, \boldsymbol{B}$, arrows). As specificity controls, deletion mutants for both GluRIID and GluRIIE (see below) were used. No in situ signals were detected here, proving full specificity for our in situ signals of both GluRIID and GluRIIE.

throughout embryonic and larval development (Fig. 1D,F). Thus, the mRNA expression pattern of the newly identified subunits appears very similar to that of GluRIIA, GluRIIB, and GluRIIC/III (Petersen et al., 1997; Marrus et al., 2004).

\section{In terms of amino acid sequence, GluRIID and GluRIIE define a new type of muscle-expressed glutamate receptor subunit}

The muscle-expressed glutamate receptor subunits of Drosophila that we identified were further analyzed. We first made sure that these proteins encode all structural features generally conserved in glutamate receptor subunits. Particularly, all putative transmembrane domains were in positions typical for glutamate receptors (Fig. 2A). As noted previously (Marrus et al., 2004), GluRIIC/III is closely related to GluRIIA and GluRIIB. We find also that GluRIID and GluRIIE are very closely related to one another (Fig. 2A,B). However, GluRIID and GluRIIE are distant from GluRIIA, GluRIIB, and GluRIIC/III. (Fig. 2B). In fact, GluRIID and GluRIIE are slightly more similar to human kainate receptor GluR6 than they are to the GluRIIA, GluRIIB, and GluRIIC/III group (Fig. 2B, dendrogram). Thus, GluRIID and GluRIIE represent a "new type" of glutamate receptor subunit that is expressed in the Drosophila muscle together with the GluRIIA, GluRIIB, and GluRIIC/III type. In sequence alignments, several other Drosophila non-NMDA glutamate receptors group in between these two clusters represented by GluRIID, GluRIIE and GluRIIA, GluRIIB, GluRIIC/III (Littleton and Ganetzky, 2000). GluRIID and GluRIIE as well as GluRIIA, GluRIIB, and GluRIIC/III all have direct orthologs in other Drosophila species (our unpublished observation), indicating that the differentiation of insect muscle-expressed glutamate receptor subunits into two structurally different groups is a conserved trait.
GluRIID is localized in postsynaptic densities of neuromuscular synapses

To study the expression of muscle-expressed glutamate receptor subunits at the subcellular level, specific antibodies were produced. Our antibody directed against a GluRIIC/III-specific peptide recognizes a single band of approximately the predicted 109 $\mathrm{kDa}$ in Drosophila embryo extracts (Fig. 3A, right lane). Moreover, the antibody labels the PSDs of all neuromuscular synapses (Fig. 3B), as shown recently with an independently produced antibody (Marrus et al., 2004). So far, GluRIIE-specific antibodies could not be obtained, mainly attributable to the fact that there are few immunogenic peptides specific for GluRIIE that are not also present within the highly related GluRIID. The specific C-terminal sequence of GluRIID (Fig. 2 A, boxed area), however, allowed the production of a specific antiserum. This antiserum recognizes SF9-cell-expressed GluRIID but not the SF9-cellexpressed GluRIIE (Fig. 3A, left lanes). When probing Drosophila embryo extracts with this GluRIID antibody, a single band of approximately the predicted $102 \mathrm{kDa}$ could be detected (Fig. 3A). In immunofluorescence stainings, GluRIID is found at all NMJs of larvae (Fig. 3C) and embryos (see below). Furthermore, the expression of GluRIID is confined to typical punctae (Fig. 3C, arrowheads). These punctae correspond to individual PSDs, because they are surrounded by the HRP staining known to have perisynaptic expression (Sone et al., 2000). Moreover, these punctae are found directly opposite to the presynaptic Nc82 label. The Nc82 monoclonal antibody labels the presynaptic active zone (Heimbeck et al., 1999; Wucherpfennig et al., 2003). We thus conclude that GluRIID specifically localizes to the PSD region of individual synaptic sites at the Drosophila NMJ. Consistently, GluRIID colocalizes substantially with the receptor subunits GluRIIB and GluRIIA on the level of individual PSDs (Fig. 3D).

\section{Null mutants of either GluRIID or GluRIIE are embryonic lethal}

To genetically investigate what role GluRIID and GluRIIE play in NMJ glutamatergic transmission, we required specific mutants for each of the two genes. We recognized that, within a collection of piggyBac transposon lines that recently became available (Thibault et al., 2004), a line with an insertion in the GluRIID locus ( $\mathrm{pBac}\{\mathrm{RB}\}^{\mathrm{e} 01443}$ ) was included. We verified that, in this line, the transposon has integrated directly into intron 6 of GluRIID (Fig. 4). Real-time PCR on embryonic cDNA showed that downstream of the transposon insertion site the GluRIID mRNA was no longer expressed, which must interfere with productive translation of GluRIID. In fact, no GluRIID protein was observed any longer at NMJs of these embryos (see below). We refer to this allele as GluRIID ${ }^{\mathrm{e} 01443}$.

For GluRIIE, excision mutagenesis screening was performed using transposon line EP28753, whose insertion site is located only 150 bp downstream of the GluRIIE gene (Fig. 4). We could recover a deficiency (GluRIIE ${ }^{E 1}$ ) (see Material and Methods), in which the genomic sequence encoding the C-terminal part of GluRIIE including the last transmembrane domain is specifically deleted. This was also verified using real-time PCR. GluRIIE ${ }^{E 1}$ does not extend into neighboring genes. Both GluRIID ${ }^{\mathrm{e} 01443}$ and $G_{\text {GluRIIE }}^{E 1}$ are embryonic lethal when homozygous and when over deficiency GluRIID\&IIE $E^{E 3}$ (Fig. 4), which deletes GluRIID and GluRIIE. GluRIID ${ }^{\mathrm{e} 01443}$ is fully viable over GluRIIE ${ }^{E 1}$ but lethal over independently retrieved null alleles of GluRIID [see companion paper by Featherstone et al. (2005) in this issue], whereas GluRIIE E1 is fully viable over these alleles. Both, GluRIID ${ }^{\mathrm{e} 01443}$ and GluRIIE E1 
A

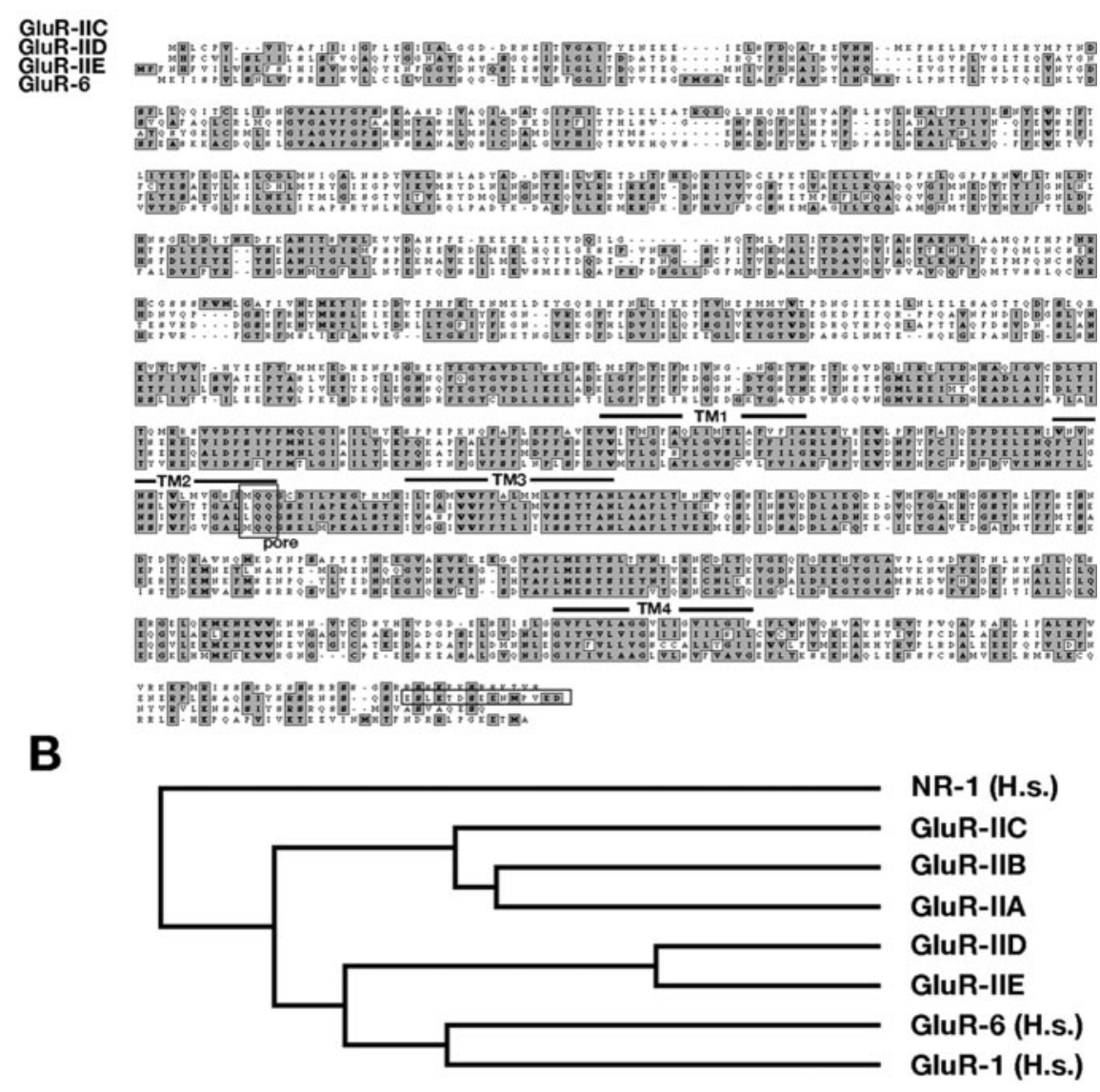

Figure 2. Sequence analysis of GluRIIID and GluRIIE. $A$, Sequence alignment of predicted amino acid sequences of (from top to bottom) GluRIIC/III, GluRIID, GluRIIE, and human kainate receptor subunit GluR6; similar amino acids are indicated by shaded boxes. Putative transmembrane domains (TM1-TM4), the channel pore region, and the C-terminal peptide of GluRIID, which was used for immunization, are indicated as well. $\boldsymbol{B}$, Dendrogram analysis comparing muscle-expressed glutamate receptor subunits of Drosophila, together with AMPA receptor subunit GluR1, kainate receptor subunit GluR6, and NMDA receptor subunit NR1 [all Homo sapiens (H.s.)]. All muscle-expressed glutamate receptor subunits of Drosophila fall into the kainate/AMPA class of glutamate receptors. However, GluRIIA, GluRIIB, and GluRIIC/III on one side and GluRIID and GluRIIE on the other side are structurally far distant. The dendrogram was generated using MacVector software Accelrys (San Diego, CA).

could be rescued to adult vitality by transgenic addition of a genomic construct encoding GluRIID or GluRIIE, respectively. However, neither the GluRIID mutant nor the GluRIIE mutant could be rescued by the respective other transgene. Thus, we obtained specific null mutants for both GluRIID and GluRIIE. Although the neighboring loci GluRIID and GluRIIE encode very similar proteins with primarily overlapping expression, both of them are essential for embryonic survival.

\footnotetext{
Interdependency between all glutamate receptor subunits for receptor function and synaptic receptor expression

Although apparently fully developed, both GluRIID or GluRIIE mutant embryos showed no coordinated movements and did not hatch. Both mutants were thus subjected to closer inspection using immunofluorescence stainings of late embryo body-wall preparations. As shown by staining against HRP and Nc82 epitope (Fig. 5A), NMJs with approximately normal amounts of release sites still formed in both GluRIID and GluRIIE mutant embryos. The same phenotype as observed in GluRIID and GluRIIE null mutants (a fully developed embryo unable of coordinated movements) is also observed within GluRIIC/III single or GluRIIA \& IIB double null mutant embryos. HRP and Nc82 cola-
}

beling showed that, also in these mutants, NMJs formed. We also colabeled with antibodies against receptor subunit GluRIIC/III. As expected, GluRIIC/III signal was absent from GluRIIC/III null mutant embryos, as well as from GluRIIA\&IIB double-mutant embryos, consistent with the idea that GluRIIC/III is an obligatory subunit that associates with either GluRIIA or GluRIIB to form glutamate receptors (Marrus et al., 2004). Surprisingly, however, the GluRIIC/III signal was also absent from the NMJs of both GluRIID or GluRIIE mutant embryos (Fig. 5A). In contrast, precise excisions of the transposons used to produce our GluRIID and GluRIIE null mutants gave wild-type-like staining of all receptor subunits (data not shown).

Based on this finding, we decided to systematically investigate the relationships between all muscle-expressed glutamate receptor subunits using the subunitspecific mutants together with the specific antibodies available in the moment (Fig. $5 B)$. NMJ staining of GluRIID was found to be absent not only from GluRIID but also from GluRIIE as well as GluRIIC and GluRIIA\&IIB mutant embryos. Vice versa, GluRIIC, GluRIIB, and GluRIIA are all absent from the NMJs of GluRIID, GluRIIE, GluRIIC, and GluRIIA\&IIB null mutant embryos. Quantitative analysis of confocal images (data not shown) did indicate a complete loss of receptor subunit expression at the NMJs of all null mutant embryos shown in Figure 5B.

As mentioned, no GluRIIE-specific antibody could be retrieved so far. Thus, GFP-labeled GluRIIE was transgenically expressed. GluRIIE ${ }^{\text {GFP }}$ specifically labels the PSDs when expressed in wild-type background. When expressed in GluRIID null background, however, PSD localization of GluRIIE ${ }^{\text {GFP }}$ is abolished (Fig. 5 C).

Thus, at the embryonic NMJ, the synaptic expression is interdependent between all essential glutamate receptor subunits. It should be emphasized, however, that all other glutamate receptor subunits are still expressed in either the GluRIIA or the GluRIIB single mutant (Marrus et al., 2004). This suggests that, at the Drosophila NMJ, the glutamate receptor is formed by four essential glutamate receptor subunits: GluRIIC, GluRIID, and GluRIIE together with either GluRIIA or GluRIIB.

Consistent with the need for GluRIID in recruiting all other subunits to the NMJ synapses, the accompanying paper by Featherstone et al. (2005) directly shows that GluRIID null mutant embryos lack any glutamate-mediated current at their NMJs using embryonic patch-clamp recordings. For GluRIIE null mutant embryos, no embryonic recordings were done so far. However, as far as we can tell, the NMJ phenotypes of GluRIIE null mutants and GluRIID null mutants are identical (no expression of glutamate receptor subunits including GluRIID; lack of coordinated embryonic mobility). Thus, it appears very likely that GluRIIE is also crucial for forming the postsynaptic glutamate receptor complex. This in turn would be essential for neurotransmission 


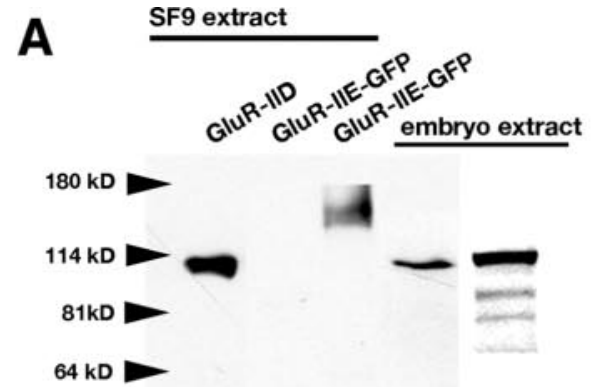

probe:
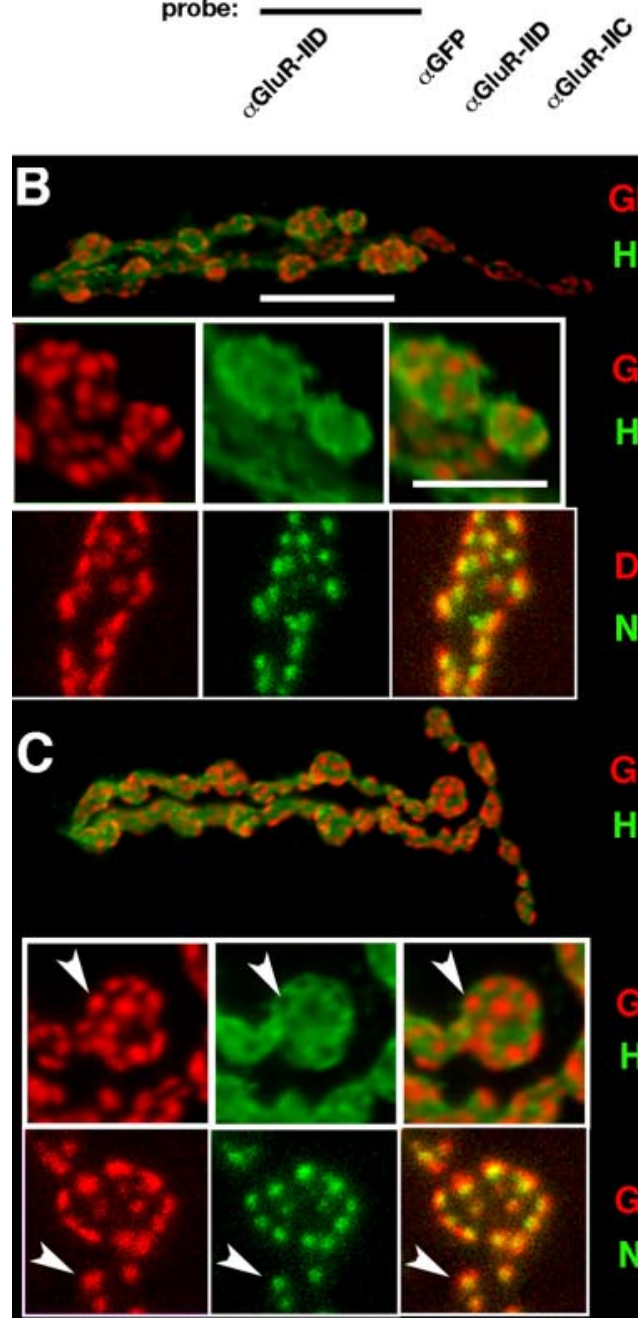

GluR-IIC

HRP

GluR-IIC

HRP

DGIuR-\|C

Nc82

GIuR-IID

HRP

GluR-IID

HRP

GluR-IID

Nc82

D

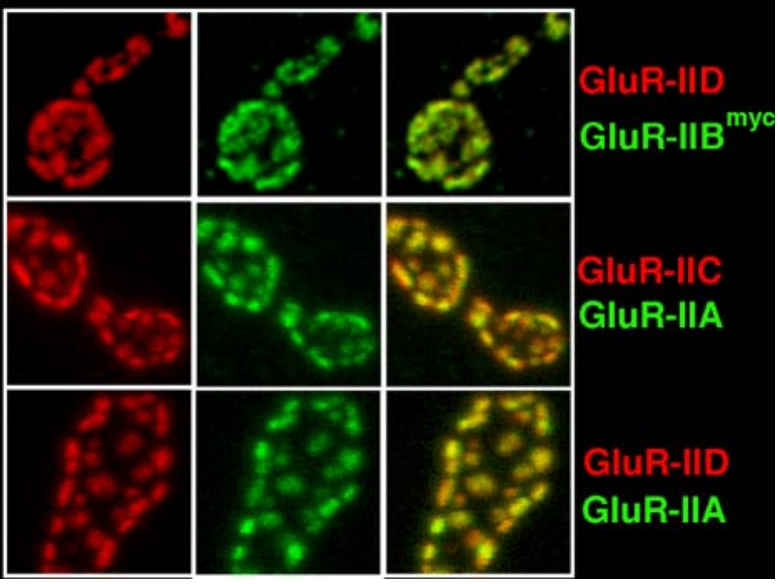

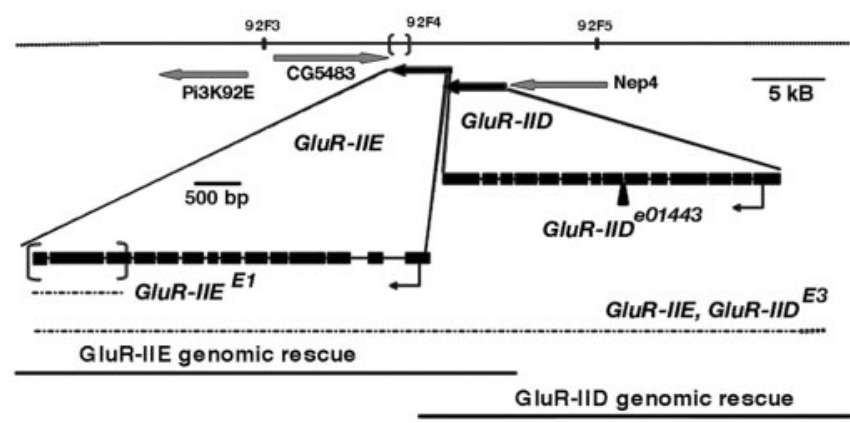

Figure 4. Genetic analysis of GluRIID and GluRIIE. GIURIID and GluRIIE map to position 92F on chromosome III. Exon-intron structure of both loci is shown; exons are boxed. The null allele GluRIID ${ }^{\mathrm{e} 01443}$ is based on a piggyBac transposon insertion within the open reading frame of the GluRIID locus. Null allele GluRIIE ${ }^{E 1}$ lacks C-terminal sequence of the protein including the last transmembrane domain of the receptor subunit. The genomic stretches used for genomic rescue constructs are shown below.

at the developing embryonic NMJ, explaining the lack of any coordinated movements in the GluRIIE null mutant embryos.

In mammals, non-NMDA glutamate receptors are apparently composed of rather closely related subunits (Wenthold et al., 1996; Mulle et al., 2000). Our genetic analysis of the subunits constituting the Drosophila NMJ glutamate receptor complex, however, provides evidence that hetero-tetrameric glutamate receptors can form by incorporating very distantly related subunits. Thereby, either GluRIIA or GluRIIB is incorporated as a fourth subunit. Although the synaptic expressions of GluRIIA and GluRIIB are not identical between individual PSDs (Marrus et al., 2004), we suggest that the amounts of GluRIIC and GluRIID should reflect the sum of GluRIIA and GluRIIB containing receptor complexes together. Consistently, the ratio between GluRIIA and either GluRIID or GluRIIC seemingly differs somewhat between individual PSDs (Fig. 3D). Because the antibodies for GluRIIC and GluRIID both derive from rabbit, no direct colabeling between these subunits could be performed.

A stoichiometric relationship between all essential subunits for synaptic expression and glutamate receptor function If the glutamate receptor does in fact form from four different subunits, the expression level of all these subunits must be able to become rate limiting for glutamate receptor formation. Thus, after partially suppressing the level of a single such subunit, glutamate receptor formation and thus synaptic expression of the respective other essential subunits should be lowered accordingly. Using muscle-specific, transgene-mediated RNA interference against GluRIIE, we could lower GluRIIE mRNA expression down to $\sim 20 \%$ without changing the mRNA expression of all other muscle-expressed subunits (real-time PCR data). These an-

\footnotetext{
Figure 3. GluRIID is expressed within postsynaptic densities. $\boldsymbol{A}$, Western blot analysis: the anti-IID peptide antibody recognizes SF9-cell-expressed GluRIID and endogenous GluRIID from Drosophila embryo extract. It does not cross-react with the related GluRIIE protein, which is SF9-cell expressed as a GFP fusion and is recognized using anti-GFP antibody with the predicted size of $\sim 145 \mathrm{kDa}$. GluRIIC/III is recognized with our peptide antibody in Drosophila embryo extract with predicted size as well. $\boldsymbol{B}, \boldsymbol{C}$, Shown are epifluorescence pictures (top 2 panels in $\boldsymbol{B}$, $\boldsymbol{C}$ ) and confocal pictures (bottom panels in $\boldsymbol{B}, \boldsymbol{C}$ ) of receptor subunits GluRIIC/III ( $\boldsymbol{B}$, red) and GluRIID ( $C$, red) together with the perisynaptic marker HRP (top panels in $B, C$, green) or active zone marker Nc82 (bottom panels in $\boldsymbol{B}, \boldsymbol{C}$, green) on third-instar NMJs of muscle 4. Scale bars: top, $8 \mu \mathrm{m}$; bottom, $4 \mu \mathrm{m}$. D. Colabeling between GluRIID and myc-tagged GluRIIIB expressed with Mhc-Gal4 driver (top), GluRIIC and GluRIIIA (middle), and between GluRIID and GluRIIA (bottom).
} 


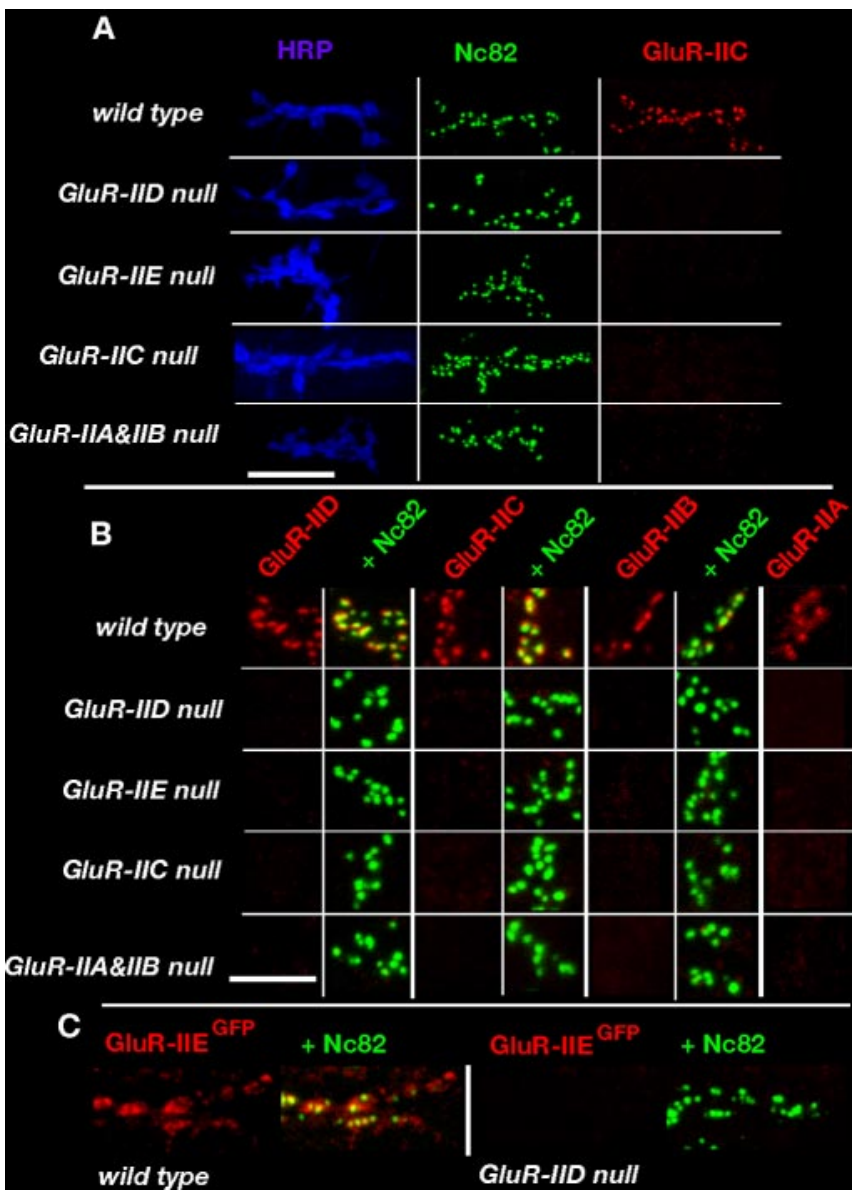

Figure 5. Interdependence between glutamate receptor subunits for NMJ expression. $\boldsymbol{A}$, Confocal images of NMJs in wild-type or in embryos null mutant for GluRIID, GluRIIE, GluRIIC, or both GluRIIA and GluRIIB (20 - 22 h old), stained with antibodies against GluRIIC/III (red), Nc82 (green), and HRP (blue). Nc82 staining indicates differentiation of active sites at the mutant NMJs. Synaptic expression of all glutamate receptor subunits fails at the mutant NMJs. Scale bar, $10 \mu \mathrm{m}$. $\boldsymbol{B}$, Higher magnifications of confocal stainings similar to $\boldsymbol{A}$. Stainings of GluRIID, GluRIIC/III, and GluRIIB are shown together with Nc82, GluRIIA alone. Scale bar, $5 \mu \mathrm{m}$. C, GFP-labeled GluRIIIE was expressed in embryonic muscles using Mhc-Gal4 driver in either wildtype background or GluRIID null background; also shown are anti-GFP, Nc82 colabeling from embryonic NMJs.

imals no longer die as embryos (as the GluRIIE null mutants do) but develop into mature larvae. At the NMJs of larvae with reduced GluRIIE, GluRIID and GluRIIC/III were clearly reduced (Fig. $6 B, D$ ). For a second experiment, we used the fact that already minimal amounts of GluRIID [produced by the "leaky" expression typically mediated by UAS-cDNA transgenes in the absence of a galactosidase-4 (Gal4)-driver line] can rescue GluRIID null mutant embryos (Fig. $6 E-H$ ). At larval NMJs of these GluRIID null mutant larvae rescued by leaky expression of GluRIID, the levels of all other glutamate receptor subunits were very strongly reduced as well (Fig. $6 H$ ). Reexpressing GluRIID to normal levels by using the muscle-specific Gal4-driver line myosin heavy chain $(M h c)$-Gal4 restored the localization of all other glutamate receptor subunits (data not shown). We conclude that the levels of GluRIID and GluRIIE within the muscles directly control the amount of glutamate receptors able to localize at the NMJ. We next asked whether the amounts of muscle GluRIIA, GluRIIB, and GluRIIC/III can reciprocally control NMJ levels of GluRIID and GluRIIE. For this, a genetic situation in which GluRIIB was fully absent and GluRIIA was simultaneously suppressed below
RNAi:

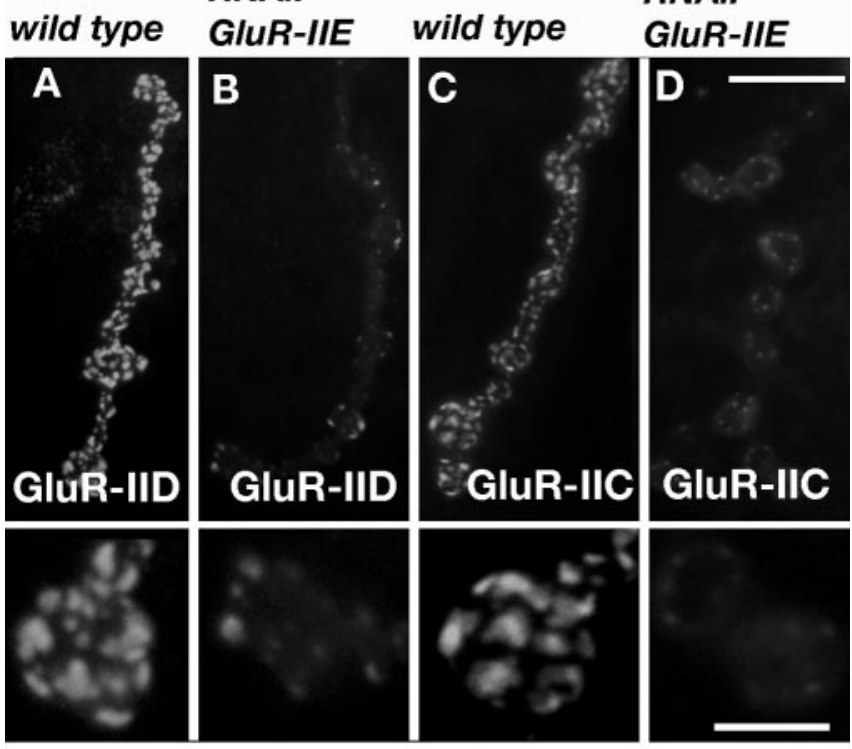

UAS-GIUR-IID / + ; GIUR-IID ${ }^{\text {null }}$
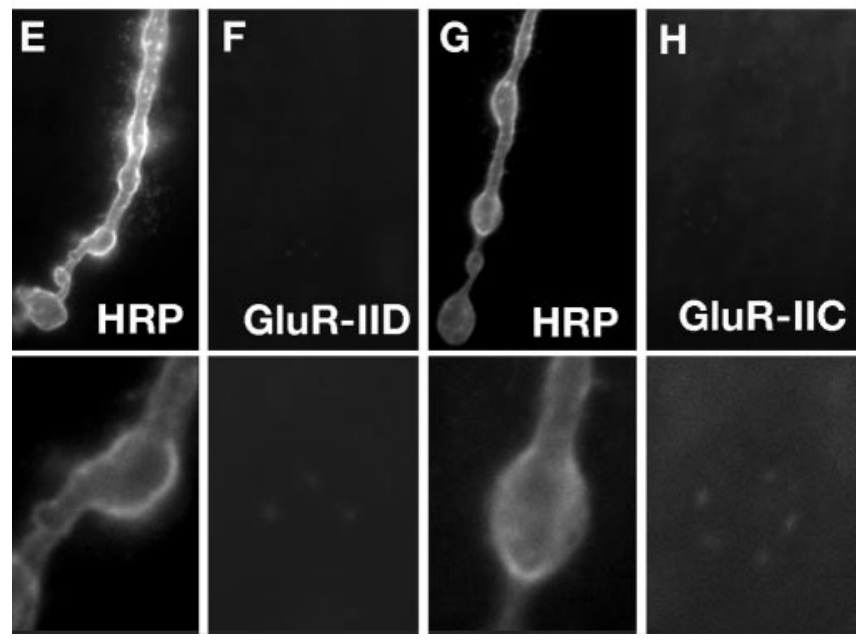

Figure 6. A partial reduction of either GluRIIE or GluRIID provokes a significant reduction of all glutamate receptor subunits at the NMJ. $A-D$, Larval NMJs (muscle 4 , abdominal segment 2) stained for GluRIIID $(\boldsymbol{A}, \boldsymbol{B})$ or GluRIIC/III $(\boldsymbol{C}, \boldsymbol{D})$ in control larvae $(G 14-G a / 4 /+; \boldsymbol{A}, \boldsymbol{C})$ or larvae experiencing muscle-specific RNA interference against GluRIIE (G14-Gal4/+; UAS-GluRIIE$R N A i ; \boldsymbol{B}, \boldsymbol{D})$. Suppression of GluRIIE leads also to a reduction in the NMJ expression of GluRIIC/III and GluRIIID. Bottom pictures represent higher magnifications. E-H, Shown are NMJs (muscle 4, abdominal segment 2) of GluRIID null mutant larvae rescued with a single copy of UAS-GluRIID

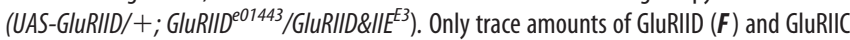
$(\boldsymbol{H})$ are expressed at the NMJ; HRP stainings $(\boldsymbol{E}, \boldsymbol{G})$ are added to show position of the NMJ. Nonlinear contrast was used to make the trace amounts of GluRIID $(\boldsymbol{F})$ and GluRIIC $(\boldsymbol{H})$ visible. Scale bars: top, $7.5 \mu \mathrm{m}$; bottom, $2.5 \mu \mathrm{m}$.

$5 \%$ of normal mRNA level (GluRIIA ${ }^{\text {hypo }}$, GluRIIB ${ }^{\text {null }}$ ) (see Material and Methods) was used. Here, the level of both GluRIIC/III and GluRIID were drastically reduced as well (Fig. $7 B, D$ ). Collectively, these data suggest that the availability of all essential glutamate receptor subunits controls receptor formation and, as expected, that assembling the proper receptor is a precondition for NMJ localization of the subunits. This seems true during both initial formation and further development of NMJs, in which many new synapses get added (Schuster et al., 1996; Gramates and Budnik, 1999). We finally asked whether glutamate receptor function would parallel our findings based on receptor immunostainings. Between the different ge- 

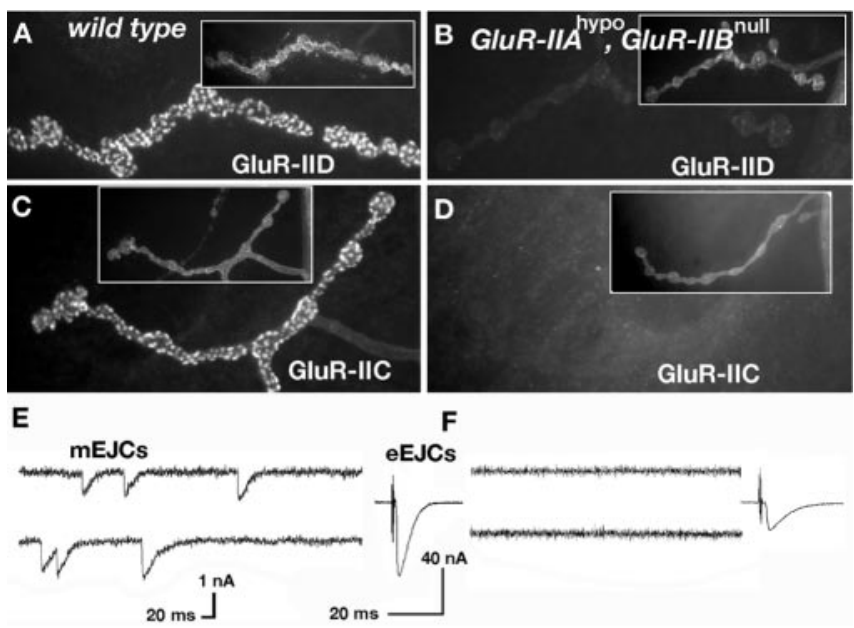

Figure 7. Minimal amounts of GluRIIA and no GluRIIB: expression of all glutamate receptor subunits and postsynaptic sensitivity are strongly reduced. $\boldsymbol{A}-\boldsymbol{D}$, Shown are third-instar larval NMJs (muscle 4, abdominal segment 2) stained for GluRIID $(\boldsymbol{A}, \boldsymbol{B})$ or GluRIIC/III $(\boldsymbol{C}, \boldsymbol{D})$ in wild-type rescued controls $(A, C$ ) or in animals having only $\sim 5 \%$ GluRIIA and no GluRIIB (GluRIIA ${ }^{\text {hypo }}$, GluRIII ${ }^{\text {null }}$ ) (see Materials and Methods). NMJ morphology in HRP labeling is shown in insets to allow NMJ visualization independent of receptor label. In GluRIIA ${ }^{\text {hypo }}$, GluRIIB ${ }^{\text {null Iarvae, }}$ the synaptic localization of both GluRIID and GluRIIC/III is strongly reduced. Apparent differences between residual GluRIID and GluRIIC/III are likely attributable to slightly different sensitivity of our antibodies. $\boldsymbol{E}, \boldsymbol{F}$, Two-electrode voltage-clamp recordings on third-instar larvae (muscle 6, abdominal segment 2), measuring spontaneous mEJCs and eEJCs. The GluRIIA ${ }^{\text {hypo }}$, GluRIIB $^{\text {null }}$ mutant animals $(\boldsymbol{F})$ are compared with wild-type controls $(\boldsymbol{E})$. Postsynaptic glutamate sensitivity is dramatically reduced in GluRIIA ${ }^{\text {hypo }}$, GluRIIB ${ }^{\text {null }}$ mutant larvae, expressed in the lack of any recordable spontaneous currents. In 15 larvae (each recording lasting several minutes), no single spontaneous response could be recorded in GluRIIA ${ }^{\text {hypo }}$, GluRIIB ${ }^{\text {null }}$ mutant larvae using two-electrode voltage-clamp configuration.

netic situations we used to partially deprive glutamate receptor subunits in larvae, GluRIIE interference produced a more moderate downregulation (Fig. $6 B, D$ ), whereas the most severe loss of glutamate receptors was observed in GluRIIA ${ }^{\text {hypo }}$, GluRIIB ${ }^{\text {null }}$ larvae (Fig. $7 F, H)$, with the latter genotype expressing clear signs of paralysis. GluRIIA $^{\text {hypo }}$, GluRIIB ${ }^{\text {null }}$ larvae were thus subjected to two-electrode voltage-clamp recordings (Fig. $6 E, F$ ). In fact, spontaneous mEJCs were below the detection limit, indicating an extreme drop of postsynaptic glutamate receptor function. Accordingly, eEJCs were also low at these NMJs (wild-type rescue, $61 \mathrm{nA}, n=12$; GluRIIA ${ }^{\text {hypo }}$, GluRIIB $^{\text {null }}, 21 \mathrm{nA}, n=11 ; p<0.00005$, Mann-Whitney, two-sided nonparametric test). We thus conclude that strongly reducing the expression of a single glutamate receptor subunit is sufficient in severely reducing the level of functional glutamate receptors at the NMJ. In summary, forming functional glutamate receptors at the Drosophila NMJ can be controlled by all five glutamate receptor subunits so far identified. Consistently, Featherstone et al. (2005) in fact demonstrate the complete absence of glutamate receptor function in GluRIID null mutant embryos by patch-clamp recordings of embryonic muscle cells.

\section{Discussion}

\section{Novel glutamate receptor subunits are essential for} glutamatergic transmission at neuromuscular synapses Glutamatergic neuromuscular synapses of Drosophila, in terms of ultrastructure and molecular composition similar to the glutamatergic synapses of our brains, have been intensely used to study synaptic function in vivo. At these synapses, three non-NMDAtype glutamate receptor subunits (GluRIIA, GluRIIB, and GluRIIC/III) have been described previously. Whereas only GluRIIA\&IIB double mutants but not the respective single mu- tants lack transmission (Petersen et al., 1997; DiAntonio et al., 1999), lack of GluRIIC/III alone is fatal with a phenotype, suggesting the absence of neurotransmission at neuromuscular synapses (Marrus et al., 2004). Doubts remained about the subunit composition of synaptic receptors at the NMJ. Here we describe two additional non-NMDA glutamate receptor subunits (GluRIID and GluRIIE) that, on the basis of in situ hybridization (Fig. 1) and antibody staining (Fig. 3), we show to be specifically expressed at the postsynaptic site of all neuromuscular synapses alongside GluRIIA, GluRIIB, and GluRIIC/III. Although GluRIID and GluRIIE are genomic neighbors and very similar to each other, they are not particularly related to the GluRIIA,IIB; IIC/III group. We could retrieve specific null mutations for both GluRIID and GluRIIE. Both GluRIID and GluRIIE null embryos, although apparently fully developed, do not hatch and are incapable of any coordinated movement. In the case of GluRIIA\&IIB as well as in the GluRIIC/III single mutant, this paralyzed phenotype was attributed to a fatal failure of synaptic transmission as well (Petersen et al., 1997). The companion paper (Featherstone et al., 2005) directly demonstrates that no postsynaptic sensitivity to glutamate is present at GluRIID null mutant embryonic muscles. Thus, GluRIID and GluRIIE are critically important for mediating glutamate-gated ionic currents at the postsynaptic site of neuromuscular synapses. It appears therefore likely that GluRIIA\&IIB double mutants as well as GluRIIC/III single mutants also lack glutamate-gated ionic current.

Together, lack of GluRIIC/III, GluRIID, and GluRIIE or of both GluRIIA and GluRIIB together leads to a fatal lack of glutamate receptor function at the embryonic NMJ. This is likely directly caused by the loss of respective subunits within the embryonic muscles because of the following arguments. First, GluRIIA, GluRIIB, GluRIIC/III, and GluRIIE expression is seemingly restricted to the somatic muscles (Petersen et al., 1997; DiAntonio et al., 1999). Second, the paralysis phenotype of these null mutants could always be fully rescued by a muscle-specific reexpression of the corresponding cDNAs (DiAntonio et al., 1999). Third, muscle-specific downregulation of GluRIIE (via RNA interference) could effectively downregulate synaptic expression of GluRIID and GluRIIC/III as well.

\section{Implications for the stoichiometry of the Drosophila glutamate receptor}

Recent studies suggested that AMPA receptors form as a tetramer of subunits (Laube et al., 1998; Mano and Teichberg, 1998; Rosenmund et al., 1998). We find that the synaptic expression of all glutamate receptor subunits is abolished after eliminating either GluRIID, GluRIIE, or GluRIIC alone or GluRIIA and GluRIIB together (Fig. 5) [see companion paper by Featherstone et al. (2005) in this issue]. Quantitative real-time PCR occluded changes of mRNA abundance as causative for glutamate receptor subunits being absent in mutants of other subunits (data not shown). Thus, an inability to join into proper receptor complexes should cause the complete absence of NMJ expression in these situations.

Together, all data indicate that essential glutamate receptor subunits (GluRIIC/III, GluRIID, and GluRIIE) are obligatory part of all postsynaptic glutamate receptors. In addition, having either GluRIIA or GluRIIB seems obligate for receptor formation. One likely scenario therefore is that the glutamate receptor population at the Drosophila NMJ is a mix of (IIA)(IIC/III) (IID)(IIE) receptors with (IIB)(IIC/III)(IID)(IIE) receptors. Tetrameric structure of glutamate receptors was suggested solely on the basis of ligand-binding studies and crystal structures of 
ligand-binding domains. An unequivocal determination of the number of subunits in a functional glutamate receptor thus still awaits physical methods that probe the structure of the receptor itself. Thus, pentameric (or higher) stoichiometry cannot be refuted in the moment and in fact was suggested in the past several times (Premkumar et al., 1997). Therefore, for example, stoichiometries as $(A)_{2}(C)(D)(E),(B)_{2}(C)(D)(E)$, or $(A)(B)(C)(D)(E)$ might still have to be considered.

GluRIIA- and GluRIIB-containing receptor complexes differ strongly in their biophysical properties, with GluRIIAcontaining receptors showing slow desensitization and GluRIIBcontaining receptors showing fast desensitization (DiAntonio et al., 1999). Thus, GluRIIA- and GluRIIB-containing complexes are likely to play different roles in synaptic function and development. In fact, increased postsynaptic GluRIIA protein levels or reduced GluRIIB gene copy number resulted in an increased strength of NMJ transmission and an addition of junctional boutons harboring increased numbers of synapses. These phenotypes were suppressed by overexpression of GluRIIB (Sigrist et al., 2002). Thus, GluRIIC/III, GluRIID, and GluRIIE might establish a "receptor platform," whereas the incorporation of either the GluRIIA or the GluRIIB subunit could determine the specific functions of the respective glutamate receptor.

As discussed above, the interdependency between all essential subunits observed for synaptic localization is easiest explained by assuming the existence of common glutamate receptor. It might, however, be argued that eliminating a certain glutamate receptor subunit could provoke an early defect during the developmental setup of neuromuscular synapses. This in turn might then interfere with the localization of other glutamate receptor complexes. This way, the genetic elimination of a certain subunit could also provoke the loss of synaptic expression (and thus also synaptic function) of another subunit, even if these two subunits would not be contained in the same glutamate receptor. At this juncture, such a scenario appears unlikely because of the following arguments. First, partial suppression of any essential glutamate receptor subunit always provokes a corresponding downregulation of glutamate receptor at larval neuromuscular synapses (Figs. 6, 7) (Featherstone et al., 2005, their Fig. 6) (Marrus and DiAntonio, 2004; Marrus et al., 2004). This suggests that all essential subunits obey a stoichiometric relationship, in which the availability of the least abundant subunit defines the overall amount of glutamate receptors and that this relationship exists throughout development. Second, Featherstone et al. (2005) convincingly demonstrate a complete loss of glutamate-mediated currents on GluRIID mutant embryonic muscles. However, extrasynaptic glutamate receptors have been directly demonstrated on wildtype embryonic muscle membranes using the very same experimental setup (Broadie and Bate, 1993) [see companion paper by Featherstone et al. (2005) in this issue]. Thus, elimination of GluRIID abolishes both all of the extrasynaptic as well as synaptic populations of glutamate receptors on embryonic muscles. Therefore, if interactions between independently forming glutamate receptors are involved, they would have to be absolutely essential already during the intracellular transport and assembly of glutamate receptors. Because we know that at least GluRIID is clearly expressed in substantial amounts within postsynaptic densities, the existence of subunits evolved only to mediate the intracellular transport or assembly of glutamate receptor complexes appears rather unlikely. However, it should be emphasized that our conclusions so far are based on determining synaptic expression and function in combination with the use of specific mutants. Unequivocal proof of our model also has to await bio- chemical analysis, ideally from the endogenously expressed glutamate receptor complexes.

\section{In vivo analysis of glutamate receptor assembly and mobility using a genetically fully accessed set of subunits}

Collectively, our in vivo analysis clearly favors the idea that forming non-NMDA glutamate receptors can depend on four different subunits. To our knowledge, a "strictly hetero-tetrameric stoichiometry" has so far not been described for other ionotropic glutamate receptors. This finding thus potentially indicates that, in vivo, some glutamate receptors can have a rather fixed subunit stoichiometry reminiscent of the stoichiometry of other types of ligand-gated ion channels as, for example, the nicotinic acetylcholine receptor (Colquhoun and Sivilotti, 2004).

Dissecting the molecular details underlying the functional assembly and cell-surface trafficking of various types of glutamate receptors is a focus of current research. It has been suggested that, in AMPA receptors, the $\mathrm{N}$-terminal domain is primarily responsible for subtype recognition among different subunits, whereas the transmembrane region and the C-terminal part of S2 are critical determinants for the formation of functional channels (Leuschner and Hoch, 1999; Ayalon and Stern-Bach, 2001). In this context, it will be interesting to explore which sequences within these the Drosophila NMJ subunits confer the essential character of individual subunits, capitalizing on the complete set of mutants for all five relevant glutamate receptor subunits now available. Given the close structural relationship between GluRIID and GluRIIE, analyzing Intersubunit chimeras between these subunits in either a GluRIID or GluRIIE mutant background might be particularly informative. The companion paper by Featherstone et al. (2005) reports that GluRIID is also expressed in the CNS of late embryos/first-instar larvae. In this context, we find that GluRIID but not GluRIIE message is expressed within heart precursor cells (Fig. $1 A$, arrowheads). Thus, between different tissues, subunit composition of glutamate receptors can overlap without being identical. It will be interesting to work out whether tissue-specific control mechanisms execute assembly and transport, reflecting the glutamate receptor subunit spectrum expressed. Moreover, our laboratory recently managed to directly visualize glutamate receptor dynamics within living intact larvae during synapse formation (our unpublished observations), applying photobleaching and photoactivation procedures in vivo. It will be very interesting, whether in fact all essential glutamate receptor subunits show coherent dynamics as to be assumed if they are within one complex.

\section{References}

Ayalon G, Stern-Bach Y (2001) Functional assembly of AMPA and kainate receptors is mediated by several discrete protein-protein interactions. Neuron 31:103-113.

Bellen HJ (1998) The fruit fly: a model organism to study the genetics of alcohol abuse and addiction? Cell 93:909-912.

Bredt DS, Nicoll RA (2003) AMPA receptor trafficking at excitatory synapses. Neuron 40:361-379.

Broadie K, Bate M (1993) Activity-dependent development of the neuromuscular synapse during Drosophila embryogenesis. Neuron 11:607-619.

Colquhoun D, Sivilotti LG (2004) Function and structure in glycine receptors and some of their relatives. Trends Neurosci 27:337-344.

DiAntonio A, Petersen SA, Heckmann M, Goodman CS (1999) Glutamate receptor expression regulates quantal size and quantal content at the Drosophila neuromuscular junction. J Neurosci 19:3023-3032.

Dingledine R, Borges K, Bowie D, Traynelis SF (1999) The glutamate receptor ion channels. Pharmacol Rev 51:7-61.

Featherstone DE, Broadie K (2000) Surprises from Drosophila: genetic 
mechanisms of synaptic development and plasticity. Brain Res Bull 53:501-511.

Featherstone DE, Rushton E, Rohrbough J, Liebl F, Karr J, Sheng Q, Rodesch CK, Broadie K (2005) An essential Drosophila glutamate receptor subunit that functions in both central neuropil and neuromuscular junction. J Neurosci 25:3199-3208.

Gramates LS, Budnik V (1999) Assembly and maturation of the Drosophila larval neuromuscular junction. Int Rev Neurobiol 43:93-117.

Heimbeck G, Bugnon V, Gendre N, Haberlin C, Stocker RF (1999) Smell and taste perception in Drosophila melanogaster larva: toxin expression studies in chemosensory neurons. J Neurosci 19:6599-6609.

Koh YH, Gramates LS, Budnik V (2000) Drosophila larval neuromuscular junction: molecular components and mechanisms underlying synaptic plasticity. Microsc Res Tech 49:14-25.

Laube B, Kuhse J, Betz H (1998) Evidence for a tetrameric structure of recombinant NMDA receptors. J Neurosci 18:2954-2961.

Leuschner WD, Hoch W (1999) Subtype-specific assembly of alpha-amino3-hydroxy-5-methyl-4-isoxazole propionic acid receptor subunits is mediated by their n-terminal domains. J Biol Chem 274:16907-16916.

Littleton JT, Ganetzky B (2000) Ion channels and synaptic organization: analysis of the Drosophila genome. Neuron 26:35-43.

Malinow R, Malenka RC (2002) AMPA receptor trafficking and synaptic plasticity. Annu Rev Neurosci 25:103-126.

Mano I, Teichberg VI (1998) A tetrameric subunit stoichiometry for a glutamate receptor-channel complex. NeuroReport 9:327-331.

Mansour M, Nagarajan N, Nehring RB, Clements JD, Rosenmund C (2001) Heteromeric AMPA receptors assemble with a preferred subunit stoichiometry and spatial arrangement. Neuron 32:841-853.

Marrus SB, DiAntonio A (2004) Preferential localization of glutamate receptors opposite sites of high presynaptic release. Curr Biol 14:924-931.

Marrus SB, Portman SL, Allen MJ, Moffat KG, DiAntonio A (2004) Differential localization of glutamate receptor subunits at the Drosophila neuromuscular junction. J Neurosci 24:1406-1415.

Mayer ML, Armstrong N (2004) Structure and function of glutamate receptor ion channels. Annu Rev Physiol 66:161-181.

Mulle C, Sailer A, Swanson GT, Brana C, O'Gorman S, Bettler B, Heinemann SF (2000) Subunit composition of kainate receptors in hippocampal interneurons. Neuron 28:475-484.

Petersen SA, Fetter RD, Noordermeer JN, Goodman CS, DiAntonio A (1997) Genetic analysis of glutamate receptors in Drosophila reveals a retrograde signal regulating presynaptic transmitter release. Neuron 19:1237-1248.

Premkumar LS, Qin F, Auerbach A (1997) Subconductance states of a mutant NMDA receptor channel kinetics, calcium, and voltage dependence. J Gen Physiol 109:181-189.

Rosenmund C, Stern-Bach Y, Stevens CF (1998) The tetrameric structure of a glutamate receptor channel. Science 280:1596-1599.

Saitoe M, Schwarz TL, Umbach JA, Gundersen CB, Kidokoro Y (2001) Absence of junctional glutamate receptor clusters in Drosophila mutants lacking spontaneous transmitter release. Science 293:514-517.
Schorge S, Colquhoun D (2003) Studies of NMDA receptor function and stoichiometry with truncated and tandem subunits. J Neurosci 23:1151-1158.

Schuster CM, Ultsch A, Schloss P, Cox JA, Schmitt B, Betz H (1991) Molecular cloning of an invertebrate glutamate receptor subunit expressed in Drosophila muscle. Science 254:112-114.

Schuster CM, Davis GW, Fetter RD, Goodman CS (1996) Genetic dissection of structural and functional components of synaptic plasticity. I. Fasciclin II controls synaptic stabilization and growth. Neuron 17:641-654.

Seeburg PH (1993) The TINS/TiPS Lecture. The molecular biology of mammalian glutamate receptor channels. Trends Neurosci 16:359-365.

Sigrist SJ, Thiel PR, Reiff DF, Lachance PE, Lasko P, Schuster CM (2000) Postsynaptic translation affects the efficacy and morphology of neuromuscular junctions. Nature 405:1062-1065.

Sigrist SJ, Thiel PR, Reiff DF, Schuster CM (2002) The postsynaptic glutamate receptor subunit DGluRIIA mediates long-term plasticity in Drosophila. J Neurosci 22:7362-7372.

Sone M, Suzuki E, Hoshino M, Hou D, Kuromi H, Fukata M, Kuroda S, Kaibuchi K, Nabeshima Y, Hama C (2000) Synaptic development is controlled in the periactive zones of Drosophila synapses. Development 127:4157-4168.

Sprengel R, Aronoff R, Volkner M, Schmitt B, Mosbach R, Kuner T (2001) Glutamate receptor channel signatures. Trends Pharmacol Sci 22:7-10.

Stewart BA, Atwood HL, Renger JJ, Wang J, Wu CF (1994) Improved stability of Drosophila larval neuromuscular preparations in haemolymphlike physiological solutions. J Comp Physiol [A] 175:179-191.

Sun Y, Olson R, Horning M, Armstrong N, Mayer M, Gouaux E (2002) Mechanism of glutamate receptor desensitization. Nature 417:245-253.

Swan LE, Wichmann C, Prange U, Schmid A, Schmidt M, Schwarz T, Ponimaskin E, Madeo F, Vorbruggen G, Sigrist SJ (2004) A glutamate receptor-interacting protein homolog organizes muscle guidance in Drosophila. Genes Dev 18:223-237.

Tautz D, Pfeifle C (1989) A non-radioactive in situ hybridization method for the localization of specific RNAs in Drosophila embryos reveals translational control of the segmentation gene hunchback. Chromosoma 98:81-85.

Thibault ST, Singer MA, Miyazaki WY, Milash B, Dompe NA, Singh CM, Buchholz R, Demsky M, Fawcett R, Francis-Lang HL, Ryner L, Cheung LM, Chong A, Erickson C, Fisher WW, Greer K, Hartouni SR, Howie E, Jakkula L, Joo D, et al. (2004) A complementary transposon tool kit for Drosophila melanogaster using P and piggyBac. Nat Genet 36:283-287.

Wenthold RJ, Petralia RS, Blahos J, II, Niedzielski AS (1996) Evidence for multiple AMPA receptor complexes in hippocampal CA1/CA2 neurons. J Neurosci 16:1982-1989.

Wucherpfennig T, Wilsch-Brauninger M, Gonzalez-Gaitan M (2003) Role of Drosophila Rab5 during endosomal trafficking at the synapse and evoked neurotransmitter release. J Cell Biol 161:609-624. 